\section{LEER PARA EDUCAR}

Presentamos a los y las lectoras de ESPIRAL, Revista de Docencia e Investigación la sección Leer para educar. En ella encontrará una guía de la producción bibliográca del Centro de Estudios en Educación en una suerte de invitación a conocer las creaciones de las autoras que, sin duda, constituyen un valioso compendio de textos para orientar y humanizar las prácticas educativas, investigativas y creativas de todos aquellos lectores que acepten leer los libros aquí reseñados.

\title{
LA CONVIVENCIA SIN VIOLENCIA. RECURSOS PARA EDUCAR
}

Nicte de Fátima Guajardo Concha ${ }^{1}$

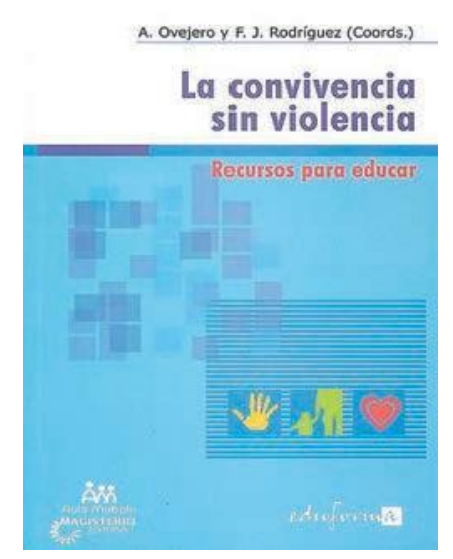

El ser humano por naturaleza es un ser social, lo que incluye la convivencia con otras personas y tal como lo mencionan los autores en la presentación del libro, en el transcurrir de la cotidianidad, el individuo se ve obligado a enfrentarse continuamente con diversos problemas, esto lo lleva a buscar recursos que le permitan encontrar una solución. De ello se trata la convivencia y el primer espacio que tienen los jóvenes para aprender a convivir es el hogar, pero también hay que reconocer que en muchos de ellos no se otorga la oportunidad de hacerlo porque no se les enseña cómo aprender a comunicarse, y creo que muchos de los problemas de convivencia que existen hoy en día se debe indudablemente a la falta de comunicación entre padres e hijos, maestros y alumnos, así como entre los mismos jóvenes.

Los autores del libro afirman que los jóvenes son la necesidad de ahora, son el mañana, y por lo mismo se convierten en los herederos de una cultura formada en el amor o en

1 Profesora del Centro de Estudios en Educación y miembro de ESPIRAL-Grupo Interdisciplinario Educativa de la Universidad Santo Tomás, Bucaramanga. el odio, en la creación o en la destrucción, en el dar o en el negar, en el compromiso o en la indiferencia; son las generaciones de adultos las responsables de preocuparse por preparar la sociedad que las nuevas generaciones recibirán y es aquí donde este libro se convierte en la oportunidad de conocer, de aprender pero sobre todo de implementar estrategias de corrección y modificación de conductas con la intención de que el día de mañana los padres y maestros no nos sintamos culpables de ver en lo que se han convertido nuestros jóvenes.

El primer capítulo "El proceso de aprendizaje social en el joven", comienza por aclarar que antes del siglo $X X$ no se hablaba ni se entendía a la adolescencia como una etapa del desarrollo del ser humano, fue hasta después de la industrialización cuando adquirió importancia la educación de los jóvenes, en ese momento es que la escolarización se convierte en obligatoria. Los autores indican a los padres y maestros que para entender a los adolescentes es indispensable tomar en cuenta el marco social y cultural de los jóvenes, por ello dan a conocer las perspectivas acerca del desarrollo humano y del proceso de socialización. Para llevar a cabo la formación y/o educación de los adolescentes existe una herramienta básica, nos referimos a la comunicación, tanto padres como profesores debemos lograr que ellos nos tengan conanza para acercarse a nosotros 
y poder entender así su desarrollo, aunado a esto debemos tener autoridad para establecer reglas y enseñar a cumplirlas, esto no confronta la disciplina así nosotros mismos ayudaremos a lograr que las capacidades de los jóvenes se desarrollen en perfecta armonía dando pautas a la formación de una personalidad con sentido común en esta hora histórica que vivenciamos.

En el segundo capítulo "Habilidades necesarias para una educación ecaz", los autores buscan que tanto los profesores y padres de familia entiendan que la educación y crianza de los jóvenes es al mismo tiempo un arte y una ciencia, el reto se halla en lograr combinar ambos factores; por lo que es indiscutible que todos aquellos que tenemos en nuestras manos la formación de los jóvenes tengamos presente que es necesaria una capacitación, lo que nos ayudará a comprender cuales son los elementos necesarios para hacer un buen trabajo.

En el tercer capítulo "Guía de recursos educativos para una socialización competente. El programa", los autores nos facilitan diversas herramientas que permitirán tanto a padres como a profesores aprender a enseñar pautas de comportamientos a los jóvenes, así como a desarrollar las habilidades básicas para poder interactuar con ellos, en otras palabras estas habilidades nos ayudarán aprender a comunicarnos; esto debido a que somos (padres y educadores) los principales agentes que ayudarán a los adolescentes a desenvolver las competencias socializadoras que dotarán a los jóvenes de los recursos necesarios para que se relacionen de forma adecuada con el medio y sepan responder a las necesidades, a los problemas y a las dudas que se le presenten. Sin duda, el apoyo afectivo es indispensable para generar la conanza en uno mismo ya que esto ayudará a prever problemas posteriores. En el texto encontramos diversas pautas que permiten el desarrollo del apoyo afectivo entre las que podemos mencionar las habilidades de comunicación, habilidades de interacción con jóvenes y el comportamiento de los educandos.

Finalmente el cuarto capítulo "Guía didáctica de recursos educativos para una socialización competente. Aplicación a grupos", está estructurado en forma de guía conformada por recursos aplicables a grupos, tiene doce sesiones cada una de ellas programada a efectuarse en más o menos noventa minutos. El esquema general de cada una de las sesiones está comprendido por los siguientes elementos: repaso, cuestionario de evaluación inicial, objetivos, desarrollo, puesta en práctica y resumen. Es importante mencionar que el desarrollo de cada una de las sesiones se complementa con diversas actividades las cuales se encuentran al nal del libro como anexos.

Como podrá comprobar el lector, el libro ofrece a profesores, padres, psicólogos, pedagogos, educadores la oportunidad de encontrar respuestas pero también preguntas acerca de cómo educar en la convivencia. Leerlo es tener la oportunidad de aprender a convivir en la familia, en la escuela y en la sociedad en general, además nos permite reexionar sobre la importancia que tiene la no violencia tanto en los centros educativos como al interior de las familias.

Reseña:

Rodríguez Díaz, Fco. Javier y Ovejero Bernal, Anastasio (Coords.) (2008). La convivencia sin violencia. Recursos para educar, colección aula múltiple, Editorial Magisterio, Colombia. 


\section{FORMAR LA IDENTIDAD TERRENAL A PARTIR DE LA LITERATURA INFANTIL}

\section{Brenda Isabel López Vargas}

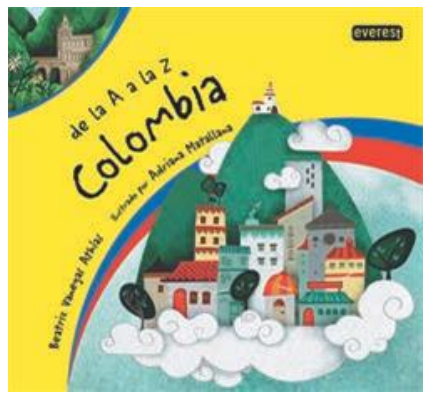

Educar para la ciudadanía desde una perspectiva compleja que supere los atavismos decimonónicos que tradicionalmente han caracterizado la educación cívica, es una tarea impostergable hoy en día para quienes nos hemos comprometido con la transformación social desde las aulas, en la que la literatura tiene mucho que aportar. $Y$ cuando esta tarea se realiza con los niños y las niñas, es aún más exigente tanto para el educador o la educadora, como para el literato o la literata que tiene claro el propósito que entraña.

De la $A$ a la $Z$ por Colombia es un libro para niños y niñas, escrito por Beatriz Vanegas Athías e ilustrado por Adriana Matallana, que los hombres y las mujeres de todas las edades disfrutarán, sin duda, y que constituye una de esas herramientas fundamentales en la tarea de formar ciudadanos y ciudadanas que se piensen universales desde el amor profundo por lo propio y que asuman un compromiso con el mundo a partir del arraigo.

Se trata de un poemario tan bellamente ilustrado, que nos permite transportarnos desde la Costa Atlántica hasta el Cerro de Monserrate, desde el Desierto Wayuu hasta la Catedral de Sal de Zipaquirá, entre otros tantos trayectos, en un recorrido que evoca nuestros viajes imaginarios de la niñez y los anhelos guardados de la madurez. Hace parte de una colección de la editorial Everest Internacional en la que se muestra lo más representativo de la historia, la naturaleza y la cultura de los países de Amé- rica Latina, a partir de palabras que empiezan con cada una de las letras del abecedario, que luego se incorporan a los versos de breves poemas. Del mismo modo que otros libros infantiles como Niños de América, de la colección Ñandú de la editorial chilena Amanuta, De la $A$ a la $Z$ Colombia maniesta claramente el propósito de acercar a los niños y las niñas a los elementos esenciales del patrimonio cultural latinoamericano, mediante una na combinación de recursos grácos y literarios.

Nunca tuvimos un vehículo más cómodo y más placentero para viajar y enamorarnos de Colombia que los poemas de Beatriz Vanegas Athías, poeta reconocida con el Premio Nacional de Poesía de la Universidad Externado de Colombia, el Premio Fondo Mixto de Poesía del Ministerio de Cultura de Sucre y el Premio Internacional de Poesía Pilar Paz Pasamar, otorgado en Cádiz, España. Sus versos cantarines y sus rimas juguetonas son, también, estrategia didáctica formidable para aprender sobre las particularidades locales que hacen tan universal la música, la literatura, la gastronomía y las tradiciones colombianas.

En ello radica el valor de esta obra literaria para los educadores y educadoras, en la posibilidad de abordar desde la estética de la poesía y de la expresión plástica de las ilustraciones, las relaciones entre lo local y lo universal, entre lo propio y lo ajeno, entre lo cercano y lo lejano, entre lo habitual y lo inhabitual, entre el yo y el nosotros, entre la diferencia y la semejanza. Se trata, en $\mathrm{n}$, de un hermoso modo de formar para la identidad terrenal que tanto necesitamos para empezar a dar ese giro humano, lógico y epistemológico que favorezca la creación de otras sociedades, de otro mundo.

Reseña:

Vanegas Athías, Beatriz (poesía) - Matallana, Adriana (ilustraciones) (2012). De la A a la Z Colombia, Editorial Everest Internacional, España. 


\section{¿QUÉ SON LAS COMPETENCIAS EN EDUCACIÓN? UNA APROXIMACIÓN DESDE EL ENFOQUE SOCIOFORMATIVO}

\section{Nicte de Fátima Guajardo Concha ${ }^{1}$}

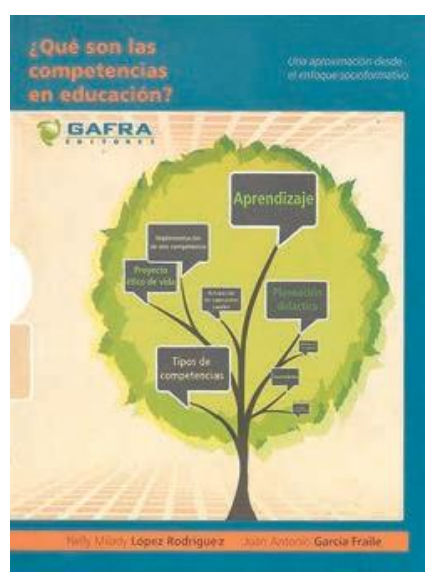

Inicio la reseña de este libro, compartiendo la denición de competencia. Éstas son entendidas como los conocimientos, habilidades y destrezas que desarrolla una persona para comprender, transformar y participar en su entorno. Las competencias no son una novedad en el ámbito educativo. Desde hace algunos años se viene hablando de este tema, y ha sido el propio Ministerio de Educación Nacional quien ha fortalecido este cambio con la única intención de que realmente exista un cambio en las concepciones de todo el que se halla inmerso en la educación.

Es necesario aclarar que existen diversos enfoques para entender que son las competencias, los autores de este libro han fundamentado toda su trayectoria investigativa es uno de ellos, nos referimos al enfoque socioformativo, el cual articula las competencias con el pensamiento complejo, la investigación acción educativa y la gestión de la calidad educativa, lo que permitirá la aplicación de diversas estrategias didácticas, así como realizar una evaluación por competencias, la cual toma en cuenta los criterios y las evidencias, todo esto con miras de realizar una verdadera educación integral de los estudiantes.

En el primer capítulo "Las competencias: concepto, tipos y retos para el educador", la intención de los autores es realizar una explicación con-

1 Profesora del Centro de Estudios en Educación y miembro de ESPIRAL-Grupo Interdisciplinario Educativa de la Universidad Santo Tomás, Bucaramanga. ceptual de los diversos enfoques (funcionalista, conductual, constructivista y socioformativo) que abordan el término competencias, partiendo de que éstas son la base para la calidad educativa. El término de competencias proviene del latín competere que signica lo que corresponde a la persona hacer con responsabilidad e idoneidad en un área y del griego agon y agonistes que quiere decir rivalizar con competición o competitividad, por último en este primer capítulo se hace mención de las diversas concepciones sobre competencias en las áreas disciplinares, así como los aportes que los ámbitos cientícos le han dado a este concepto.

Los autores ofrecen en el segundo capítulo "Las competencias: un enfoque socioformativo", una explicación amplia de lo que comprende el término de competencias desde el enfoque socioformativo, el cual permite la formación humana integral como un proyecto ético de vida, tomando en cuenta diversos desempeños, contextos, integración de saberes así como la idoneidad. Cuatro saberes son esenciales en la formación por competencias nos referimos a saber ser, saber convivir, saber conocer y saber hacer; en su conjunto se permite como ya mencionamos la formación integral debido a que toma en cuenta las siguientes dimensiones:

- Dimensión cognitiva: conocimientos, conceptos y habilidades cognitivas

- Dimensión socio-afectiva: actitudes y valores

- Dimensión actuacional: habilidades, procedimientos y técnicas.

Por lo anterior se considera que las competencias desde este enfoque son algo más que 
un simplemente saber hacer, yendo esto muy de la mano con el pensamiento complejo propuesto por Edgar Morin.

Para la construcción de una competencia son dos los pasos que se deben seguir, en un primer momento se requiere efectuar la identicación de la competencia a partir del contexto, esto se hace a través de verbo de desempeño (se dene tomando en cuenta el nivel de desarrollo de pensamiento que tiene el estudiante), objeto conceptual (son los elementos teóricos a formar), nalidad (el para que se van a formar los elementos teóricos) y condición de referencia (se toman en cuenta los criterios, códigos éticos, normativos, referentes y contextos que sirven para fundamentar los elementos teóricos); en segundo lugar se debe realizar la descripción de criterios y evidencias; los primeros se reeren a las pautas de valoración de los saberes de la competencia y las evidencias son las pruebas concretas del logro de la competencia y nos permite vericar los criterios, estos dos últimos elementos serán de gran utilidad al momento de efectuar la evaluación por competencias, la cual se efectúa a través de las matrices de evaluación para lo cual es indispensable denir los niveles de desarrollo o dominio de la competencia, los autores han establecido 5 niveles, estos son, nulo, inicial-receptivo, básico, autónomo y estratégico; cada uno de ellos tiene unas características especícas que permiten comprobar la adquisición del aprendizaje por parte del estudiante.

Se considera importante mencionar que existen tres clases de competencias, las cuales son: competencias básicas, competencias genéricas o transversales y competencias especícas, todas ellas en su conjunto buscan formar de manera integral tomando en cuenta los saberes esenciales ya mencionados.
Finalmente los autores nos ofrecen algunas de las críticas más frecuentes que se le han hecho a la formación por competencias, algunas de ellas se reeren a que las competencias no existen o que son una moda, estas críticas son bien recibidas pero el enfoque socioformativo muestra que apoyado en la literatura existente que las competencias son construcciones teóricas e integradoras que representan las diversas dimensiones del desempeño idóneo ante actividades y problemas del contexto en el que se encuentra involucrado el estudiante.

Por último se podrá encontrar al nal de la obra un glosario que contiene los conceptos esenciales y que ayuda a claricar un poco más que son las competencias desde en el enfoque socioformativo.

\section{Reseña:}

López Rodríguez, Nelly Milady y García Fraile, Juan Antonio (2012). ¿Qué son las competencias en educación? Una aproximación desde el enfoque socioformativo, Gafra Editores, México. 


\section{UN LIBRO DE MÚLTIPLES LECTURAS}

\section{Beatriz Vanegas Athías}

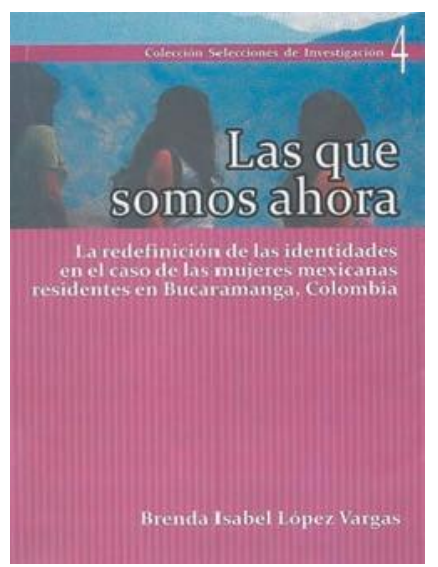

Sí, Las que somos ahora. La redenición de las identidades en el caso de las mujeres mexicanas residentes en Bucaramanga, Colombia de la investigadora Brenda Isabel López Vargas es un libro que soporta múltiples lecturas, por eso es un libro bello, profundo y necesario.

Bello, porque a lo largo de las setenta y una páginas que lo componen, la autora despliega una prosa clara y contundente necesaria ante tanto libro académico que considera lo ininteligible del lenguaje como el requerimiento ideal para alcanzar la categoría de intelectual. Es un libro gestado en el corazón de la academia pero capaz de trascender y llegar al maestro, al sociólogo, al investigador y al lector desprevenido interesado en degustar un buen libro. Las que somos ahora nos va contando desde el rigor propio del paradigma cualitativo y la consecuente técnica de recolección de datos llamada relato de vida, la integración de mujeres mexicanas por matrimonio, a la capital de Santander en Colombia.

Profundo y novedoso porque indaga cientícamente en el proceso migratorio de tres mujeres ocurrido a nivel latinoamericano, hasta reconstruir el nacionalismo mexicano a partir, como bien lo explicita la autora "del discurso, las concepciones y los comportamientos de estas mujeres en la familia y en la relación con otros grupos e instituciones sociales" (López, 2012, p.16)

Y necesario porque es toda una lección de metodología de la investigación. Dos bien estructurados capítulos se desarrollan para perlar la migración femenina mexicana por matrimonio. En el primero, la autora se aproxima a la teoría de la migración femenina través de autores como Marina Ariza, De Oliveira, Eva Jiménez, Ivonne Szas, Montserrat Guibernau y David Miller, entre otros.

En el segundo apartado se presenta el análisis de los casos de mujeres mexicanas residentes en Colombia. Aquí, conviene que el lector sepa que se encontrará con un capítulo potente por cuanto la autora alcanza un alto equilibrio cientíco que no deja Itrar subjetividades. Por el contrario, la investigadora hace de su propia experiencia, un material invaluable para alcanzar la conguración identitaria surgida de la migración de mexicanas hacia Bucaramanga, Colombia. Dicho en palabras de la investigadora: "Es importante tener en cuenta que la migración no sólo genera un replanteamiento de las identidades de los migrantes sino también una transformación paulatina de las formas de convivencia en las sociedades receptoras" (López, 2012, p. 31)

Invitamos pues a que los lectores comprueben por qué hemos calicado Las que somos ahora. La redenición de las identidades en el caso de las mujeres mexicanas residentes en $\mathrm{Bu}$ caramanga, Colombia como un texto bello, profundo y necesario.

Reseña:

López Vargas Brenda Isabel (2012). Las que somos ahora. La redenición de las identidades en el caso de las mujeres mexicanas residentes en Bucaramanga, Colombia, Editorial Universidad Santo Tomás, Bucaramanga. 
